\title{
SOME THEORETICAL AND COMPUTATIONAL ASPECTS OF THE INVERSE GENERALIZED POWER WEIBULL DISTRIBUTION
}

\author{
Mahmoud Ali Selim ${ }^{1}$ \\ ${ }^{1}$ Community College, King Khalid University, KSA \& Department of Statistics, Al-Azhar \\ University, Egypt
}

\begin{abstract}
This paper introduces a new three-parameter distribution called inverse generalized power Weibull distribution. This distribution can be regarded as a reciprocal of the generalized power Weibull distribution. The new distribution is characterized by being a general formula for some well-known distributions, namely inverse Weibull, inverse exponential, inverse Rayleigh and inverse Nadarajah-Haghighi distributions. Some of the mathematical properties of the new distribution including the quantile, density, cumulative distribution functions, moments, moments generating function and order statistics are derived. The model parameters are estimated using the maximum likelihood method. The Monte Carlo simulation study is used to assess the performance of the maximum likelihood estimators in terms of mean squared errors. Two real datasets are used to demonstrate the flexibility of the new distribution as well as to demonstrate its applicability.
\end{abstract}

Keywords: Inverse Weibull distribution, Nadarajah-Haghighi distribution, Maximum likelihood estimation, Order statistics, Monte Carlo simulation

\footnotetext{
${ }^{1}$ Selim.one@gmail.com
} 


\section{Introduction}

Weibull distribution is one of the most important distributions used in reliability engineering and other disciplines. Also, it adequately describes the observed failure times of many different types of components and phenomena. Therefore, the Weibull distribution was more widely used as a basis for several generalizations. See, for example, the exponentiated Weibull distribution by Mudholkar and Srivastava (1993), beta-Weibull distribution by Lee et al. (2007) and Kumaraswamy Weibull distribution by Cordeiro et al. (2010). For more detail on the generalizations of Weibull distribution, refer to the books by Murthy et al. (2004), Rinne (2008) and Lai (2014). Also, Haghighi and Nikulin (2006) proposed a new extension of Weibull distribution called the generalized power Weibull distribution. The cumulative density function (cdf) and probability density function (pdf) of the generalized power Weibull distribution, respectively are

$$
F(y)=1-\exp \left\{1-\left(1+\lambda y^{\theta}\right)^{\alpha}\right\}, \lambda, \alpha, \theta>0, y>0
$$

and

$$
f(y)=\lambda \alpha \theta y^{\theta-1}\left(1+\lambda y^{\theta}\right)^{\alpha-1} \exp \left\{1-\left(1+\lambda y^{\theta}\right)^{\alpha}\right\}, \lambda, \alpha, \theta>0, \quad y>0
$$

where $\alpha$ and $\theta$ are two shape parameters and $\lambda$ is a scale parameter. The Weibull distribution is a special case of (1) when $\alpha=1$. Its hazard rate function according to Nikulin and Haghighi (2009) can be constant, monotone, unimodal, bathtub-shaped. In the literature, some extensions of the generalized power Weibull distribution proposed by many authors, such as Selim and Badr (2016) proposed the Kumaraswamy generalized power Weibull distribution, Selim (2018) proposed the generalized power generalized Weibull distribution, Khan (2018) proposed the transmuted generalized power Weibull distribution and Pena-Ramirez et al. (2018) proposed The exponentiated power generalized Weibull distribution.

This paper aims to introduce a reciprocal of the generalized power Weibull distribution named inverse generalized power Weibull (IGPW) distribution and studies its mathematical properties. The motivations for deriving the inverse generalized power Weibull distribution are to provide more usefulness and flexibility of the ordinary distribution and to improve its goodness-of-fit in comparison with the well-known distributions in lifetime data analysis.

The rest of this paper is organized as follows. The inverse generalized power Weibull distribution and the special cases thereof are introduced in Section 2. Some of the mathematical properties of IGPW distribution are derived in Section 3, including the quantile function, skewness, kurtosis, ordinary moments, moment generating function and order statistics. The maximum likelihood estimation of the model parameters is introduced in Section 4. In Section 5, the Monte Carlo simulation study is used to assess the performance of the maximum likelihood estimators in terms of mean squared errors. Two real data sets are used to illustrate the usefulness of the IGPW distribution in Section 6. The final Section is devoted to the conclusion. 


\section{Inverse Generalized Power Weibull Distribution}

The inverse generalized power Weibull distribution can be derived using the transformation $X=1 / Y$, whereupon if the random variable $Y$ follows the GPW distribution, the random variable $X$ follows the IGPW distribution. The cdf and pdf of IGPW distribution are given, respectively, by

$$
\mathrm{F}(\mathrm{x})=\exp \left\{1-\left(1+\lambda x^{-\theta}\right)^{\alpha}\right\}, \alpha, \theta, \lambda>0, \quad x>0
$$

and

$$
f(x)=\alpha \theta \lambda x^{-\theta-1}\left(1+\lambda x^{-\theta}\right)^{\alpha-1} \exp \left\{1-\left(1+\lambda x^{-\theta}\right)^{\alpha}\right\}
$$

where $\lambda$ is scale parameter and $\alpha, \theta$ are shape parameters. This model has inverse Weibull (IW) distribution as a special case when $\alpha=1$. Hence, it can also be considered as an extension of the inverse exponential distribution which is developed by Keller et al. (1982) when $\alpha=\theta=$ 1. The graphs of the pdf and cdf for selected values of the model parameters are plotted in Fig. 1 .

The survival $s(x)$ and the hazard rate $h(x)$ functions of the IGPW distribution are given, respectively by

$$
s(x)=1-F(x)=1-\exp \left\{1-\left(1+\lambda x^{-\theta}\right)^{\alpha}\right\}, \quad x>0
$$

and

$$
h(x)=\frac{f(x)}{s(x)}=\frac{\alpha \theta \lambda x^{-\theta-1}\left(1+\lambda x^{-\theta}\right)^{\alpha-1} \exp \left\{1-\left(1+\lambda x^{-\theta}\right)^{\alpha}\right\}}{1-\exp \left\{1-\left(1+\lambda x^{-\theta}\right)^{\alpha}\right\}}, \quad x>0
$$

Fig. 2, shows some possible shapes of the IGPW hazard rate function.

The reversed hazard $r(x)$ and the cumulative failure rate $H(x)$ functions of the IGPW distribution are given, respectively by

and

$$
r(x)=\alpha \theta \lambda x^{-\theta-1}\left(1+\lambda x^{-\theta}\right)^{\alpha-1}, \quad x>0,
$$

$$
H(x)=\left(1+\lambda x^{-\theta}\right)^{\alpha}-1, \quad x>0 .
$$
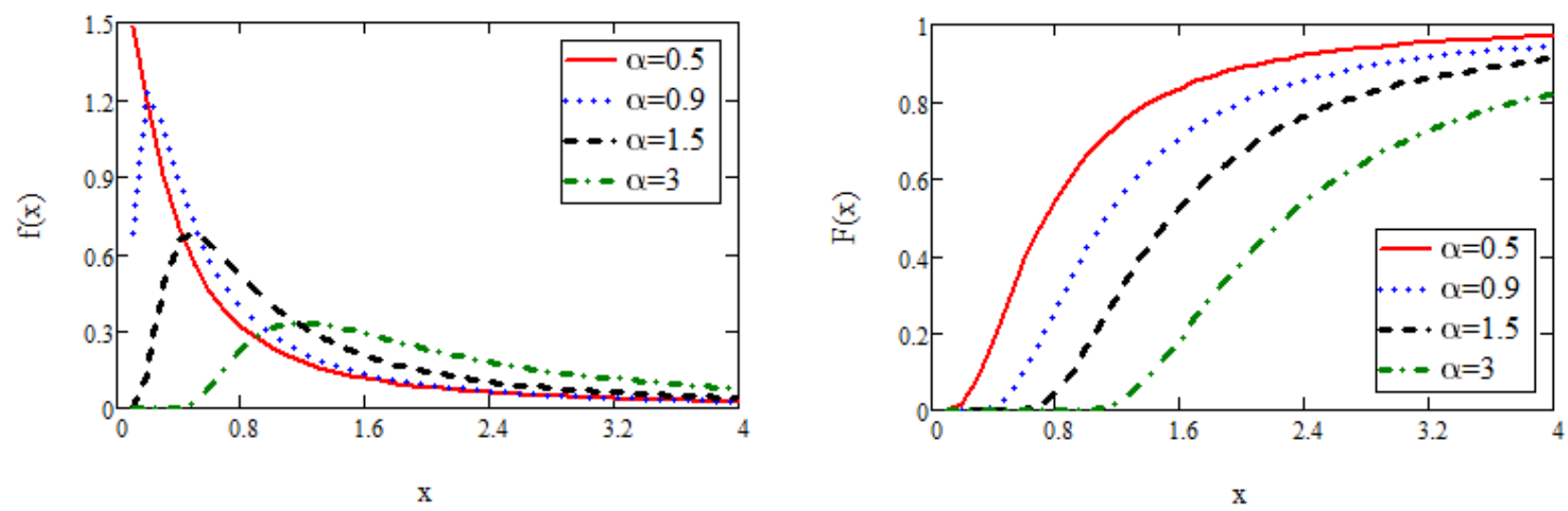

Fig. 1: Some possible shapes of the IGPW density function (left panel) and the IGPW cumulative density function (right panel) 


\subsection{Special cases of the IGPW distribution}

A number of the important distributions can be obtained as special cases of the IGPW distribution, are specifically inverse Weibull (IW), inverse exponential (IE), inverse Nadarajah-Haghighi $(I N H)$ and inverse Rayleigh $(I R)$ distributions. The special cases of IGPW distribution for selected values of the parameters $(\alpha, \theta)$ are listed in Table 1.

Table 1. Special cases of the IGPW distribution

\begin{tabular}{ccccc}
\hline Model & $\lambda$ & $\alpha$ & $\theta$ & Author $(s)$ \\
\hline$I W$ & - & 1 & - & Keller et al. (1982); $($ Keller and Kamath \\
& & & & 1982) \\
$I E$ & - & 1 & 1 & (Keller et al. 1982) \\
$I N H$ & - & - & 1 & $($ Tahir et al. 2018) \\
$I R$ & - & 1 & 2 & $($ Voda 1972) \\
\hline
\end{tabular}

\section{The Statistical Properties}

In this section, some of the statistical properties of IGPW distribution including the quantile function, random variables generation function, moments, moment generating function, skewness, kurtosis and order statistics are derived.

\subsection{Quantile function and simulation}

The quantile function has a number of important applications, for example, it can be used to obtain the median, skewness, kurtosis and can be also used to generate random variables. The q-th quantile is a solution of the following equation $F\left(x_{q}\right)=q, \quad 0 \leq q \leq 1$.
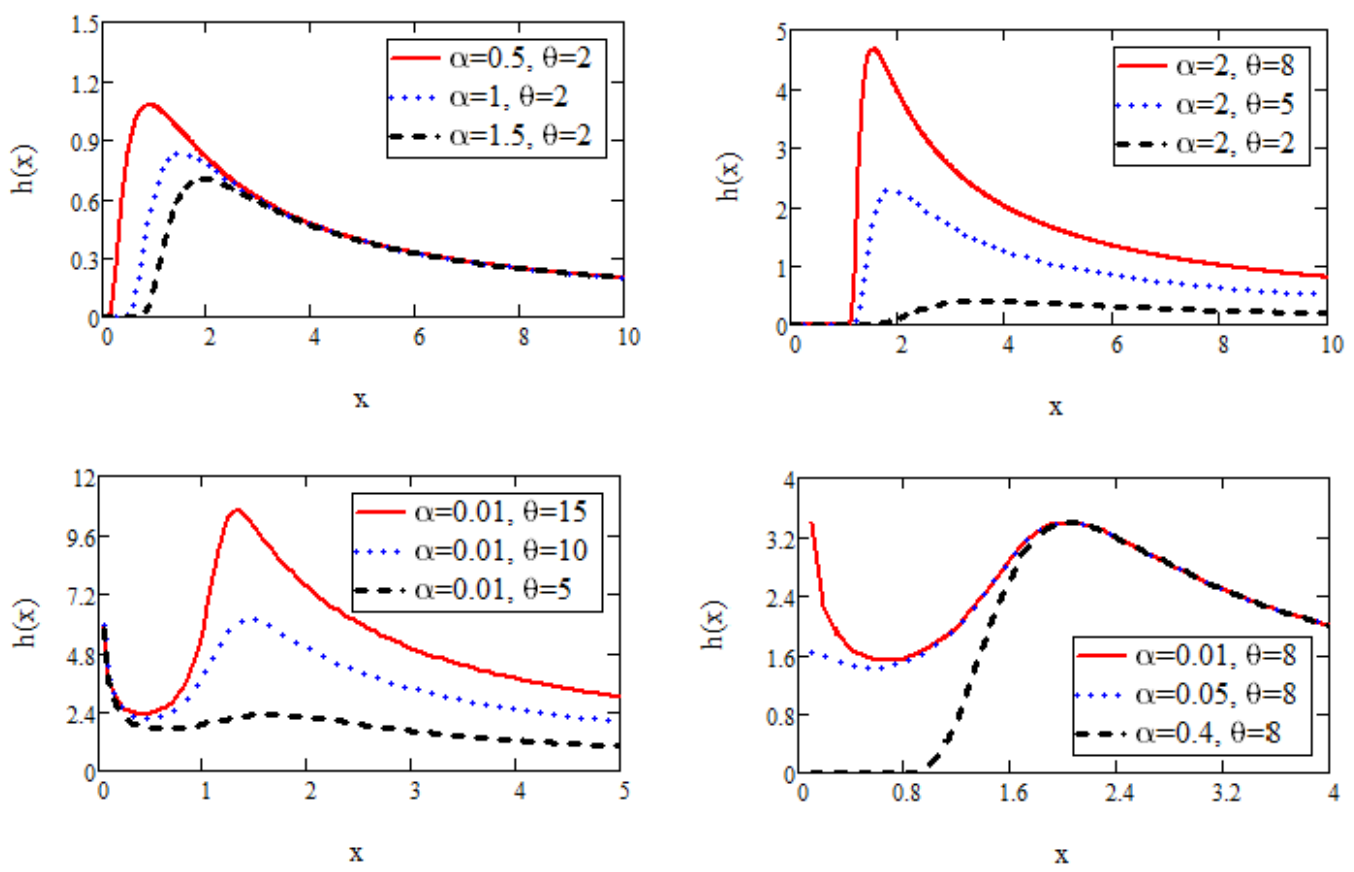

Fig. 2: Some possible shapes of the IGPW hazard rate function

Thus, the quantile function $Q(q)$ corresponding of the IGPW distribution is 


$$
Q(q)=\lambda^{1 / \theta}[\sqrt[\alpha]{1-\ln (q)}-1]^{-1 / \theta}
$$

Setting $\mathrm{q}=0.5$, in equation (9) we obtain the median of the IGPW distribution, as follows

$$
Q(0.5)=\lambda^{1 / \theta}[\sqrt[\alpha]{1-\ln (0.5)}-1]^{-1 / \theta}
$$

Fig. 3, shows the median of the IGPW distribution as a function of the parameters $\alpha$ and $\theta$.

The random variables $X$ of IGPW distribution can be simulated using equation (9) as following

$$
X=\lambda^{1 / \theta}[\sqrt[\alpha]{1-\ln (u)}-1]^{-1 / \theta}
$$

where $u \sim$ the uniform $(0,1)$ distribution and $X \sim \operatorname{IGPW}(\lambda, \alpha, \theta)$.

\subsection{Skewness and kurtosis}

The shortcomings of the classical skewness and kurtosis measures can be avoided by using the skewness and kurtosis measures based on quantiles like Bowley's skewness and Moors' kurtosis. The Bowley's skewness measure based on quartiles ((Kenney and Keeping 1962)) is given by

$$
S k=\frac{Q_{3 / 4}-2 Q_{1 / 2}+Q_{1 / 4}}{Q_{3 / 4}-Q_{1 / 4}}
$$

and the Moors' kurtosis measure based on octiles (Moors (1988)) is given by

$$
K u=\frac{Q_{7 / 8}-Q_{5 / 8}+Q_{3 / 8}-Q_{1 / 8}}{Q_{6 / 8}{ }^{-Q_{2} / 8}}
$$

The Fig. 3, shows the behaviors of median, skewness and kurtosis of the IGPW distribution as a function of the parameters $\alpha$ and $\theta$.
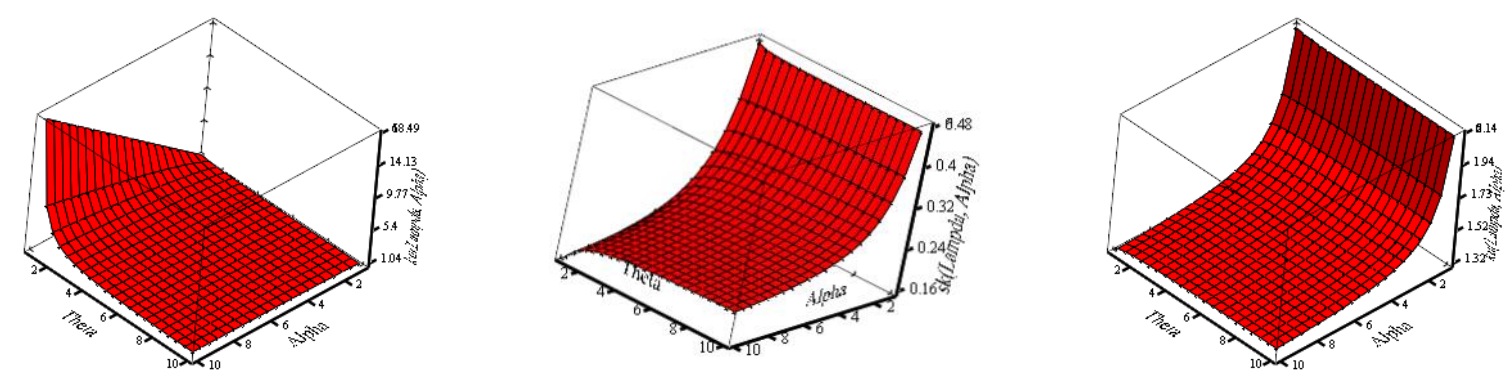

Fig. 3: The median (left panel), Skewness (middle panel) and kurtosis (right panel) of the IGPW distribution as a function of the parameters $\alpha$ and $\theta$.

\subsection{Moments and moment generating function}

The moments and moment generating function of the IGPW distribution are given by the following theorems:

Theorem 1. If $X$ has the IGPW distribution, then the $r$ th moments of $X$ for integer value of $r \theta^{-1}$ is

$$
\mu_{r}^{\prime}=e \lambda^{r \theta^{-1}} \sum_{i=0}^{-r \theta^{-1}}(-1)^{i-r \theta^{-1}}\left(\begin{array}{c}
-r \theta^{-1} \\
i
\end{array}\right) \Gamma\left(i \alpha^{-1}+1,1\right), \quad r<1
$$

where $\Gamma(\mathrm{a}, \mathrm{b})$ denotes the upper incomplete gamma function and $e$ is Euler's number.

Proof. The $r$ th moment of $X$ is defined as follows

Inserting Eq.(4) into Eq. (15), yields

$$
\mu_{r}^{\prime}=E\left(X^{r}\right)=\int_{0}^{\infty} x^{r} f(x) d x
$$




$$
\mu_{r}^{\prime}=\operatorname{e\alpha \lambda \theta } \int_{0}^{\infty} x^{r-\theta-1}\left(1+\frac{\lambda}{x^{\theta}}\right)^{\alpha-1} \exp \left\{-\left(1+\frac{\lambda}{x^{\theta}}\right)^{\alpha}\right\} d x
$$

Let $v=\left(1+\frac{\lambda}{x^{\theta}}\right)^{\alpha}$, the above expression reduce to

$$
\mu_{r}^{\prime}=e \lambda^{\frac{r}{\theta}} \int_{1}^{\infty}\left(v^{\frac{1}{\alpha}}-1\right)^{-r \theta^{-1}} e^{-v} d v
$$

Then, by applying the binomial expansion of

$$
\left(v^{\frac{1}{\alpha}}-1\right)^{-r \theta^{-1}}=\sum_{i=0}^{\infty}(-1)^{i-r \theta^{-1}}\left(\begin{array}{c}
-r \theta^{-1} \\
i
\end{array}\right) v^{\frac{i}{\alpha}}
$$

we get

$$
\mu_{r}^{\prime}=e \lambda^{r \theta^{-1}} \sum_{i=0}^{-r \theta^{-1}}(-1)^{i-r \theta^{-1}}\left(\begin{array}{c}
-r \theta^{-1} \\
i
\end{array}\right) \int_{1}^{\infty} v^{\frac{i}{\alpha}} e^{-v} d v
$$

By integrating the incomplete gamma function in (18) we get the $\mathrm{r}$ th moment of $X$ as follows

$$
\mu_{r}^{\prime}=e \lambda^{r \theta^{-1}} \sum_{i=0}^{-r \theta^{-1}}(-1)^{i-r \theta^{-1}}\left(\begin{array}{c}
-r \theta^{-1} \\
\mathrm{i}
\end{array}\right) \Gamma\left[\frac{i}{\alpha}+1,1\right]
$$

If $\alpha=\theta=1$, we get the moments of inverse exponential distribution as follows

$$
\mu_{r}^{\prime}=e \lambda^{r} \sum_{\mathrm{i}=0}^{-\mathrm{r}}(-1)^{\mathrm{i}-\mathrm{r}}\left(\begin{array}{c}
-\mathrm{r} \\
\mathrm{i}
\end{array}\right) \Gamma[\mathrm{i}+1,1]
$$

And if $\theta=1$, we get the moments of inverse Weibull distribution as follows

$$
\mu_{r}^{\prime}=e \lambda^{r \theta^{-1}} \sum_{i=0}^{-r \theta^{-1}}(-1)^{i-r \theta^{-1}}\left(\begin{array}{c}
-r \theta^{-1} \\
i
\end{array}\right) \Gamma(i+1,1), \quad r<1
$$

Theorem 2. If $X \sim I G P W$ distribution, then for any integer value of $r \theta^{-1}$, the moment generating function is

$$
M_{x}(t)=e \lambda^{r \theta^{-1}} \sum_{r=0}^{\infty} \sum_{i=0}^{-r \theta^{-1}}(-1)^{i-r \theta^{-1}}\left(\begin{array}{c}
-r \theta^{-1} \\
\mathrm{i}
\end{array}\right) \Gamma\left[\frac{i}{\alpha}+1,1\right]
$$

Proof. The moment generating function is defined as follows

$$
M_{x}(t)=\int_{0}^{\infty} e^{t x} f(x) d x
$$

Using exponential function formula $e^{t x}=\sum_{r=0}^{\infty} \frac{(t x)^{r}}{r !}$, we get

$$
M_{x}(t)=\sum_{r=0}^{\infty} \frac{t^{r}}{r !} E\left(X^{r}\right)
$$

By inserting Eq. (14) in Eq. (20), yields the moment generating function of IGPW distribution as in (19).

\subsection{Order statistics}

Assuming that $x_{(1)}, x_{(2)}, \ldots, x_{(n)}$ are the order statistics of a random sample follows a continuous distribution with cdf $F(x)$ and pdf $f(x)$, then the pdf of $X_{(k)}$ is given by

$$
f_{k: n}(x)=\frac{n !}{(k-1) !(n-k) !} f(x)[F(x)]^{k-1}[1-F(x)]^{n-k}, \quad k=1,2, \ldots, n
$$

Let $X$ is a random variable of $I G P W$ distribution, then the density function of the k-th order statistics of the $I G P W$ distribution is

$$
f_{k: n}(x)=\frac{n !}{(k-1) !(n-k) !} \alpha \theta \lambda x^{-\theta-1}\left(1+\lambda x^{-\theta}\right)^{\alpha-1}\left[e^{1-\left(1+\lambda x^{-\theta}\right)^{\alpha}}\right]^{k}\left[1-e^{1-\left(1+\lambda x^{-\theta}\right)^{\alpha}}\right]^{n-k}
$$

If $k=1$, the pdf of order statistics is 


$$
f_{1: n}(x)=n \alpha \theta \lambda x^{-\theta-1}\left(1+\lambda x^{-\theta}\right)^{\alpha-1} e^{1-\left(1+\lambda x^{-\theta}\right)^{\alpha}}\left[1-e^{1-\left(1+\lambda x^{-\theta}\right)^{\alpha}}\right]^{n-1}
$$

and if $k=\mathrm{n}$, the pdf of order statistics is

$$
f_{n: n}(x)=n \alpha \theta \lambda x^{-\theta-1}\left(1+\lambda x^{-\theta}\right)^{\alpha-1}\left[e^{1-\left(1+\lambda x^{-\theta}\right)^{\alpha}}\right]^{n}
$$

\section{Maximum Likelihood Estimation}

This section is devoted to discussing the maximum likelihood estimation $(M L E)$ and the approximate confidence intervals for the unknown parameters of $I G P W$ distribution. Let $x_{1}, x_{1}, \ldots, x_{n}$ is a complete random sample of size $\mathrm{n}$ from the IGPW distribution. Then the likelihood function (LF) is

$$
L(\alpha, \theta, \lambda \mid x)=(\alpha \theta \lambda)^{n} \prod_{i=1}^{n} x^{-\theta-1}\left(1+\lambda x^{-\theta}\right)^{\alpha-1} \exp \left\{1-\left(1+\lambda x^{-\theta}\right)^{\alpha}\right\}
$$

and the $\log$-likelihood function $(\ln L)$ is

$$
\begin{gathered}
\ln L=n(\ln (\alpha \theta \lambda)+1)-(\theta+1) \sum_{i=1}^{n} \ln x_{i}+(\alpha-1) \sum_{i=1}^{n} \ln \left(1+\lambda x_{i}^{-\theta}\right)- \\
\sum_{i=1}^{n}\left(1+\lambda x_{i}^{-\theta}\right)^{\alpha}
\end{gathered}
$$

The maximum likelihood estimators of $\alpha, \theta$ and $\lambda$ are the solution of the following three equations

$$
\begin{gathered}
\frac{\partial \ln L}{\partial \lambda}=\frac{n}{\lambda}+(\alpha-1) \sum_{i=1}^{n} \frac{1}{x_{i} \theta\left(1+\lambda x_{i}^{-\theta}\right)}-\alpha \sum_{i=1}^{n} \frac{\left(1+\lambda x_{i}^{-\theta}\right)^{\alpha-1}}{x_{i}^{\theta}}=0, \\
\frac{\partial \ln L}{\partial \alpha}=\frac{n}{\alpha}+\sum_{i=1}^{n} \ln \left(1+\lambda x_{i}^{-\theta}\right)-\sum_{i=1}^{n} \ln \left(1+\lambda x_{i}^{-\theta}\right)\left(1+\lambda x_{i}^{-\theta}\right)^{\alpha}=0 \\
\frac{\partial \ln L}{\partial \theta}=\frac{n}{\theta}-\sum_{i=1}^{n} \ln x_{i}-(\alpha-1) \lambda \sum_{i=1}^{n} \frac{\ln x_{i} x_{i}^{-\theta}}{\left(1+\lambda x_{i}^{-\theta}\right)}+\sum_{i=1}^{n} \frac{\alpha \lambda \ln x_{i}}{x_{i} \theta\left(1+\lambda x_{i}{ }^{-\theta}\right)^{1-\alpha}}=0
\end{gathered}
$$

These nonlinear equations cannot be analytically solved, but the statistical software like R program (Team (2015)) can be used to solve them numerically using iterative techniques.

The asymptotic variance-covariance matrix of the MLEs for the three parameters $\alpha, \theta$ and $\lambda$ is the inverse of the observed Fisher information matrix as follows

$$
\hat{\sigma}=\left[\begin{array}{ccc}
-\frac{\partial^{2} \ln L}{\partial \alpha^{2}} & -\frac{\partial^{2} \ln L}{\partial \alpha \partial \lambda} & -\frac{\partial^{2} \ln L}{\partial \alpha \partial \theta} \\
-\frac{\partial^{2} \ln L}{\partial \alpha \partial \lambda} & -\frac{\partial^{2} \ln L}{\partial \lambda^{2}} & -\frac{\partial^{2} \ln L}{\partial \lambda \partial \theta} \\
-\frac{\partial^{2} \ln L}{\partial \alpha \partial \theta} & -\frac{\partial^{2} \ln L}{\partial \lambda \partial \theta} & -\frac{\partial^{2} \ln L}{\partial \theta^{2}}
\end{array}\right]_{\alpha=\widehat{\alpha}, \lambda=\widehat{\lambda}, \theta=\widehat{\theta}}^{-1}=\left[\begin{array}{ccc}
\hat{\sigma}_{\alpha}^{2} & \hat{\sigma}_{\alpha, \lambda} & \hat{\sigma}_{\alpha, \theta} \\
\hat{\sigma}_{\alpha, \lambda} & \hat{\sigma}_{\lambda}^{2} & \hat{\sigma}_{\lambda, \theta} \\
\hat{\sigma}_{\alpha, \theta} & \hat{\sigma}_{\lambda, \theta} & \hat{\sigma}_{\theta}^{2}
\end{array}\right]
$$

The elements of the sample Fisher information matrix can be obtained by deriving the second derivatives of the log-likelihood function (26) and evaluating them at the MLEs ((Cohen 1965)). These elements can be derived as follow

$$
\begin{gathered}
-\frac{\partial^{2} \ln L}{\partial \alpha^{2}}=\frac{n}{\alpha^{2}}+\sum_{i=1}^{n} \ln \left(1+\lambda x_{i}^{-\theta}\right)^{2}\left(1+\lambda x_{i}^{-\theta}\right)^{\alpha} \\
-\frac{\partial^{2} \ln L}{\partial \lambda^{2}}=\frac{n}{\lambda^{2}}+(\alpha-1) \sum_{i=1}^{n} \frac{\left(1+\lambda x_{i}^{-\theta}\right)^{-2}}{x_{i}^{2 \theta}}+\alpha(\alpha-1) \sum_{i=1}^{n} \frac{\left(1+\lambda x_{i}^{-\theta}\right)^{\alpha-2}}{x_{i}^{2 \theta}} \\
-\frac{\partial^{2} \ln L}{\partial \theta^{2}}=\frac{n}{\theta^{2}}-(\alpha-1) \lambda \sum_{i=1}^{n} \frac{\ln \left(x_{i}\right)^{2} x_{i}^{-\theta}}{\left(1+\lambda x_{i}^{-\theta}\right)}+\alpha \lambda \sum_{i=1}^{n} \frac{\ln \left(x_{i}\right)^{2} x_{i}^{-\theta}}{\left(1+\lambda x_{i}^{-\theta}\right)^{1-\alpha}}+\lambda^{2}(\alpha- \\
\text { 1) } \sum_{i=1}^{n} \frac{\ln \left(x_{i}\right)^{2} x_{i}^{-2 \theta}}{\left(1+\lambda x_{i}^{-\theta}\right)^{2}}+\lambda^{2} \alpha(\alpha-1) \sum_{i=1}^{n} \frac{\ln \left(x_{i}\right)^{2} x_{i}^{-2 \theta}}{\left(1+\lambda x_{i}^{-\theta}\right)^{2-\alpha}}
\end{gathered}
$$




$$
\begin{gathered}
-\frac{\partial^{2} \ln L}{\partial \alpha \partial \lambda}=-\sum_{i=1}^{n}\left(1+\frac{\lambda}{x_{i}^{\theta}}\right)^{\alpha-1} x_{i}^{-\theta}\left[-1-\alpha \ln \left(1+\frac{\lambda}{x_{i}^{\theta}}\right)+\left(1+\frac{\lambda}{x_{i}^{\theta}}\right)^{-\alpha}\right], \\
-\frac{\partial^{2} \ln L}{\partial \alpha \partial \theta}=-\sum_{i=1}^{n} \lambda \ln x_{i}\left(1+\frac{\lambda}{x_{i}^{\theta}}\right)^{\alpha-1} x_{i}^{-\theta}\left[1+\alpha \ln \left(1+\frac{\lambda}{x_{i}^{\theta}}\right)-\left(1+\frac{\lambda}{x_{i}^{\theta}}\right)^{-\alpha}\right], \\
-\frac{\partial^{2} \ln L}{\partial \lambda \partial \theta}=-\sum_{i=1}^{n}(\alpha-1) \ln \left(x_{i}\right) x_{i}^{-\theta}\left[\alpha(\alpha-1)^{-1}\left(1+\lambda x_{i}^{-\theta}\right)^{\alpha-1}-\left(1+\lambda x_{i}^{-\theta}\right)^{-1}+\right. \\
\left.\lambda x_{i}^{-\theta}\left(1+\lambda x_{i}^{-\theta}\right)^{-2}+\alpha \lambda x_{i}^{-\theta}\left(1+\lambda x_{i}^{-\theta}\right)^{\alpha-2}\right] .
\end{gathered}
$$

The asymptotic normality of the MLE can be used to compute the approximate confidence intervals for the parameters $\alpha, \lambda$ and $\theta$ as follow

$$
\hat{\alpha}_{M L} \pm z_{\tau / 2} \sqrt{\hat{\sigma}_{\alpha}^{2}}, \quad \hat{\lambda}_{M L} \pm z_{\tau / 2} \sqrt{\hat{\sigma}_{\lambda}^{2}} \text { and } \hat{\theta}_{M L} \pm z_{\tau / 2} \sqrt{\hat{\sigma}_{\theta}^{2}}
$$

where $\mathrm{z}_{\tau / 2}$ is an upper $(\tau / 2) 100 \%$ of the standard normal distribution.

\section{Simulation Study}

In this section, the simulation study is executed to assess the performance of the proposed MLE method for estimating the parameters of IGPW distribution. Monte Carlo experiments were carried out based on generated data from IGPW distribution. By using the inversion method in Section 3.1, We generated 1000 samples of size $n=20,50,100$ from IGPW distribution for different combinations of parameters $\alpha, \lambda$ and $\theta$. The mean square errors (MSE) of the MLEs were computed using the "CG" optimization' method in R program. The simulation results were displayed in Table 2. The main conclusion from the figures in Table 2, is that the mean square errors of MLEs decrease with increasing the sample size. This indicates that the MLE method is suitable for estimating the unknown parameters of IGPW distribution. 
Table 2: Mean square errors of the MLEs' $\widehat{\alpha}, \hat{\lambda}, \hat{\theta}$

\begin{tabular}{|c|c|c|c|c|c|c|c|c|c|}
\hline Parameters & \multicolumn{3}{|c|}{$n=25$} & \multicolumn{3}{c|}{$n=50$} & \multicolumn{3}{c|}{$n=100$} \\
\hline$\alpha, \lambda, \theta$ & $\widehat{\alpha}$ & $\hat{\lambda}$ & $\hat{\theta}$ & $\widehat{\alpha}$ & $\hat{\lambda}$ & $\hat{\theta}$ & $\widehat{\alpha}$ & $\hat{\lambda}$ & $\hat{\theta}$ \\
\hline $0.5,0.5,0.5$ & 0.2708 & 0.0169 & 0.3979 & 0.0834 & 0.0162 & 0.2890 & 0.0706 & 0.0151 & 0.2621 \\
\hline $1,0.5,0.5$ & 0.9698 & 0.3349 & 0.1144 & 0.7509 & 0.2783 & 0.0868 & 0.4699 & 0.1865 & 0.0705 \\
\hline $1.5,0.5,0.5$ & 0.6715 & 0.7987 & 0.1129 & 0.2145 & 0.3303 & 0.1036 & 0.2217 & 0.3096 & 0.0410 \\
\hline $0.5,1,0.5$ & 0.0797 & 0.2063 & 0.5093 & 0.0445 & 0.1355 & 0.4508 & 0.0523 & 0.0930 & 0.4220 \\
\hline $0.5,1.5,0.5$ & 0.0772 & 0.7335 & 0.4802 & 0.0453 & 0.5282 & 0.4562 & 0.0403 & 0.3793 & 0.3218 \\
\hline $0.5,0.5,1$ & 0.1727 & 0.0142 & 0.3131 & 0.1145 & 0.0129 & 0.3044 & 0.0836 & 0.0102 & 0.1863 \\
\hline $0.5,0.5,1.5$ & 0.3400 & 0.0198 & 0.4910 & 0.2845 & 0.0168 & 0.3666 & 0.1592 & 0.0139 & 0.2545 \\
\hline
\end{tabular}

\section{Real Data Illustration}

This section illustrates the usefulness of the IGPW distribution using two real datasets. These datasets are described as follows:

\section{The data set (I): Stress-rupture life data}

The first data set consists of 76 observations of the strengths of the life of fatigue fracture of Kevlar 373/epoxy that are subject to constant pressure at the $90 \%$ stress level until all had failed. For previous studies with the data sets see Andrews and Herzberg (1985), Barlow et al. (1984) and Oluyede et al. (2016). These data are: 0.0251, 0.0886, 0.0891, 0.2501, 0.3113, $0.3451,0.4763,0.5650,0.5671,0.6566,0.6748,0.6751,0.6753,0.7696,0.8375,0.8391$, $0.8425,0.8645,0.8851,0.9113,0.9120,0.9836,1.0483,1.0596,1.0773,1.1733,1.2570$, $1.2766,1.2985,1.3211,1.3503,1.3551,1.4595,1.4880,1.5728,1.5733,1.7083,1.7263$, $1.7460,1.7630,1.7746,1.8275,1.8375,1.8503,1.8808,1.8878,1.8881,1.9316,1.9558$, $2.0048,2.0408,2.0903,2.1093,2.1330,2.2100,2.2460,2.2878,2.3203,2.3470,2.3513$, $2.4951,2.5260,2.9911,3.0256,3.2678,3.4045,3.4846,3.7433,3.7455,3.9143,4.8073$, 5.4005, 5.4435, 5.5295, 6.5541, 9.0960.

\section{The data set (II): Remission times data}

The second data set represents the remission times (in months) of a random sample of 128 bladder cancer patients (see Lee and Wang (2003)). The data are: 0.08, 2.09, 3.48, 4.87, 6.94, 8.66, 13.11, 23.63, 0.20, 2.23, 3.52, 4.98, 6.97, 9.02, 13.29, 0.40, 2.26, 3.57, 5.06, 7.09, 9.22, $13.80,25.74,0.50,2.46,3.64,5.09,7.26,9.47,14.24,25.82,0.51,2.54,3.70,5.17,7.28,9.74$, $14.76,26.31,0.81,2.62,3.82,5.32,7.32,10.06,14.77,32.15,2.64,3.88,5.32,7.39,10.34$, $14.83,34.26,0.90,2.69,4.18,5.34,7.59,10.66,15.96,36.66,1.05,2.69,4.23,5.41,7.62$, $10.75,16.62,43.01,1.19,2.75,4.26,5.41,7.63,17.12,46.12,1.26,2.83,4.33,5.49,7.66$, $11.25,17.14,79.05,1.35,2.87,5.62,7.87,11.64,17.36,1.40,3.02,4.34,5.71,7.93,11.79$, $18.10,1.46,4.40,5.85,8.26,11.98,19.13,1.76,3.25,4.50,6.25,8.37,12.02,2.02,3.31,4.51$, $6.54,8.53,12.03,20.28,2.02,3.36,6.76,12.07,21.73,2.07,3.36,6.93,8.65,12.63,22.69$. The data has been used by Kumar et al. (2015), El-Gohary et al. (2015), Chandra (2017) and De Andrade and Zea (2018).

We fitted the above-mentioned datasets using MLE to the inverse generalized power Weibull (IGPW), inverse Nadarajah-Haghighi (INH), inverse Weibull (IW) and inverse exponential (IE) distributions. The MLEs and their standard errors for IGPW, INH, IW, and IE distributions are displayed in Table 3. The fitted models were compared by using Cramér- 
von Mises $\left(\mathrm{W}^{*}\right)$, Anderson Darling $\left(\mathrm{A}^{*}\right)$, Kolmogorov-Smirnov $(K-S)$, -Log-likelihood $(-\ln L)$, Akaike Information Criterion (AIC), Consistent Akaike Information Criterion $(C A I C)$, Bayesian Information Criterion (BIC) and Hannan-Quinn Information Criterion (HQIC). Based on these criteria, the best model is the one that achieves the lowest values for the information criteria and goodness-of-fit statistics. Hence, it is clear from the numerical results in Table 4, that the IGPW model provides a better fit than the other competing models. The Figures 4 and 5 display the graphical comparison of the fitted models for datasets I and II, respectively. Also, these figures graphically illustrate that IGPW distribution provides the best fit to our data sets, as compared to the other considered models. Therefore, the IGPW model can be used as a possible alternative to the well- known models like inverse exponential and inverse Weibull models.
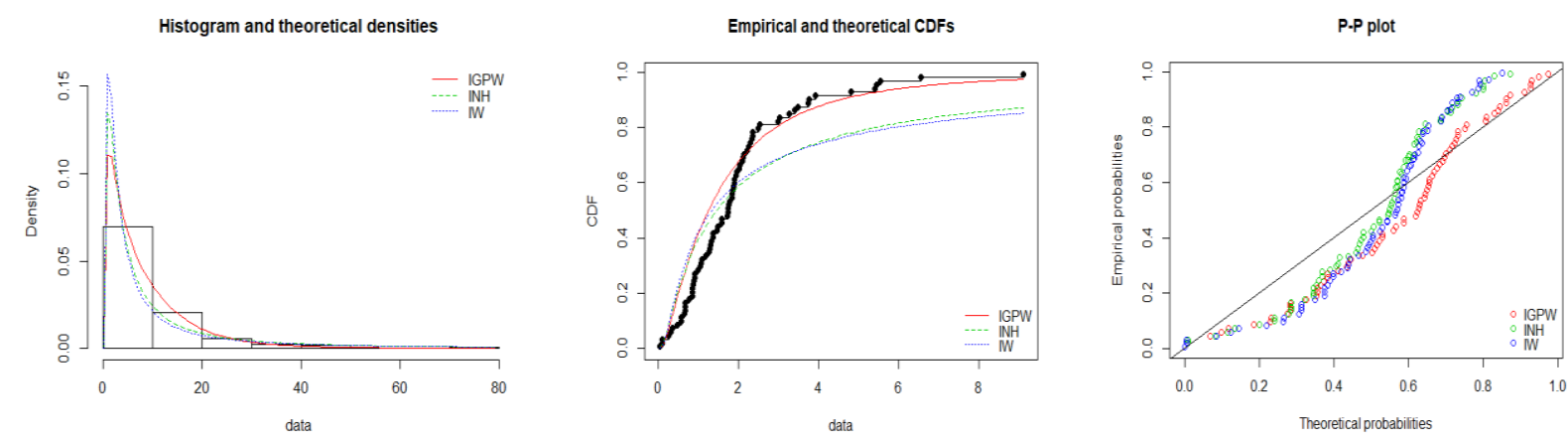

Fig. 4: Histogram and estimated densities (left panel); Theoretical and estimated CDFs (middle panel); PP plots (right panel) for stress-rupture life data
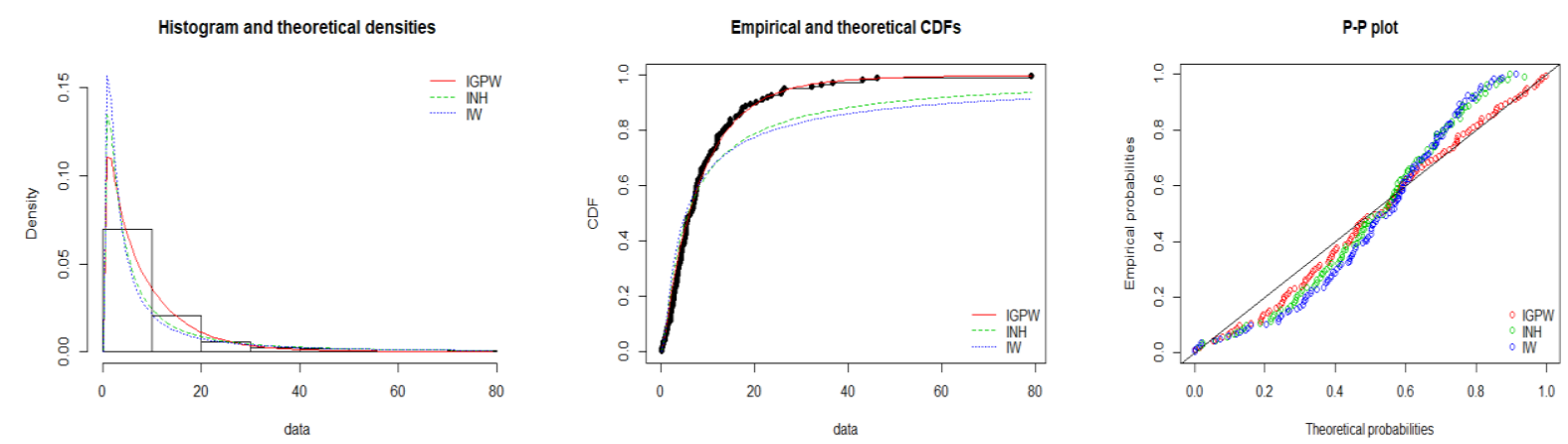

Fig. 5: Histogram and estimated densities (left panel); Theoretical and estimated CDFs (middle panel); PP plots (right panel) for Remission times data 
Table 3. The estimates and the standard errors (in parentheses) for data set I and II

\begin{tabular}{ccccc}
\hline Data set & Model & $\alpha$ & $\lambda$ & $\theta$ \\
\hline Data set I & IE & - & $0.6248(0.0716)$ & - \\
& IR & - & $0.0395(0.0045)$ & - \\
& IW & - & $0.8625(0.1089)$ & $0.7585(0.0540)$ \\
& INH & $0.5130(0.0594)$ & $2.6070(0.7020)$ & - \\
& IGPW & $0.2356(0.0327)$ & $12.3717(4.6809)$ & $2.0876(0.2692)$ \\
\hline \multirow{2}{*}{ Data set II } & IE & - & $2.4847(0.2200)$ & - \\
& IR & - & $0.6174(0.0545)$ & - \\
& IW & - & $2.4311(0.2192)$ & $0.7520(0.0424)$ \\
& INH & $0.5064(0.0480)$ & $10.5947(2.3220)$ & - \\
& IGPW & $0.4435(0.0482)$ & $15.7165(4.8326)$ & $1.2110(0.1023)$ \\
\hline
\end{tabular}

Table 4. The estimates of the goodness-of-fit test for data set I and II

\begin{tabular}{cccccccccc}
\hline & Model & K-S & $\mathrm{W}^{*}$ & $\mathrm{~A}^{*}$ & $-\mathrm{L}$ & $\mathrm{AIC}$ & $\mathrm{CAIC}$ & BIC & HQIC \\
\hline $\begin{array}{c}\text { Data set } \\
\text { I }\end{array}$ & IE & 0.2899 & 1.2059 & 6.8516 & 163.1015 & 328.203 & 328.257 & 330.5337 & 329.1344 \\
& IR & 0.7940 & 2.0476 & 10.9515 & 345.9147 & 693.8294 & 693.8834 & 696.1601 & 694.7608 \\
& IW & 0.1886 & 0.9166 & 5.3388 & 153.5393 & 311.0787 & 311.2431 & 315.7401 & 312.9416 \\
& INH & 0.1798 & 0.5652 & 3.3981 & 144.5465 & 293.0930 & 293.2574 & 297.7545 & 294.9560 \\
& IGPW & 0.1841 & 0.3082 & 1.8968 & 132.0617 & 270.1234 & 270.4568 & 277.1156 & 272.9179 \\
\hline Data set & IE & 0.2311 & 1.1139 & 6.6074 & 460.382 & 922.765 & 922.796 & 925.617 & 923.923 \\
II & IR & 0.7502 & 2.3754 & 13.2264 & 774.342 & 1550.683 & 1550.715 & 1553.535 & 1551.842 \\
& IW & 0.1408 & 0.7443 & 4.5464 & 444.001 & 892.002 & 892.098 & 897.706 & 894.319 \\
& INH & 0.1636 & 0.3565 & 2.2844 & 431.059 & 866.118 & 866.214 & 871.822 & 868.436 \\
& IGPW & 0.1364 & 0.3368 & 2.1713 & 426.910 & 859.819 & 860.013 & 868.375 & 863.296 \\
\hline
\end{tabular}




\section{Conclusion}

This paper introduces a new three-parameter distribution, called the inverse generalized power Weibull distribution. This distribution is considered as a reciprocal of the generalized power Weibull distribution and a generalization of inverse Weibull distribution. Some of the statistical properties of the inverted generalized power Weibull distribution, including the moments, hazard rate function, quantile function and order statistics are derived. The maximum likelihood method is used to estimate the model parameters. The performances of the maximum likelihood estimators are assessed in terms of mean squared errors using Monte Carlo simulation. The practical applications have established that the proposed distribution is quite useful for dealing with reliability data and behaves better than its four special cases (inverse Weibull, inverse exponential, inverse Rayleigh and inverse Nadarajah-Haghighi distributions).

\section{Acknowledgements}

The author would like to thank the Editor in Chief and the two referees for their careful reading and comments which greatly improved the presentation of the article. 


\section{References}

[1] Andrews, D., and A. Herzberg. 1985. Data: a collection of problems from many fields for the student and research worker.

[2] Barlow, R., R. Toland, and T. Freeman. 1984. A Bayesian analysis of stress-rupture life of kevlar 49/epoxy spherical pressure vessels. Paper read at Proc. Conference on Applications of Statistics', Marcel Dekker, New York.

[3] Chandra, S. S. a. N. 2017. The quasi xgamma distribution with application in bladder cancer data. Journal of Data Science 15:61-76.

[4] Cohen, A. C. 1965. Maximum likelihood estimation in the Weibull distribution based on complete and on censored samples. Technometrics 7 (4):579-588.

[5] Cordeiro, G. M., E. M. Ortega, and S. Nadarajah. 2010. The Kumaraswamy Weibull distribution with application to failure data. Journal of the Franklin Institute 347 (8):1399-1429.

[6] De Andrade, T. A., and L. M. Zea. 2018. The exponentiated generalized extended Pareto distribution. Journal of Data Science 16 (4):781-800.

[7] El-Gohary, A., A. El-Bassiouny, and M. El-Morshedy. 2015. Inverse flexible weibull extension distribution. International Journal of Computer Applications 115 (2).

[8] Haghighi, F., and M. Nikulin. 2006. A chi-squared test for power generalized Weibull family for the head-and-neck cancer censored data. Journal of Mathematical Sciences 133 (3):1333-1341.

[9] Keller, A., and A. Kamath. 1982. Alternate reliability models for mechanical systems. ESA Reliability and Maintainability p 411-415(SEE N 83-20178 10-38).

[10] Keller, A., A. Kamath, and U. Perera. 1982. Reliability analysis of CNC machine tools. Reliability Engineering 3 (6):449-473.

[11] Kenney, J., and E. Keeping. 1962. Kurtosis. Mathematics of Statistics 3:102-103.

[12] Khan, M. S. 2018. Transmuted generalized power Weibull distribution. Thailand Statistician 16 (2):156-172.

[13] Kumar, D., U. Singh, and S. K. Singh. 2015. A method of proposing new distribution and its application to Bladder cancer patients data. J. Stat. Appl. Pro. Lett 2 (3):235-245.

[14] Lai, C.-D. 2014. Generalized weibull distributions. In Generalized Weibull Distributions: Springer, 23-75.

[15] Lee, C., F. Famoye, and O. Olumolade. 2007. Beta-Weibull distribution: some properties and applications to censored data. Journal of modern applied statistical methods 6 (1):17.

[16] Lee, E. T., and J. Wang. 2003. Statistical methods for survival data analysis. Vol. 476: John Wiley \& Sons.

[17] Moors, J. 1988. A quantile alternative for kurtosis. The statistician:25-32.

[18] Mudholkar, G. S., and D. K. Srivastava. 1993. Exponentiated Weibull family for analyzing bathtub failure-rate data. IEEE Transactions on Reliability 42 (2):299-302.

[19] Murthy, D. P., M. Xie, and R. Jiang. 2004. Weibull models. Vol. 505: John Wiley \& Sons. 
[20] Nikulin, M., and F. Haghighi. 2009. On the power generalizedWeibull family: model for cancer censored data. Metron-International Journal of Statistics 67 (1):75-86.

[21] Oluyede, B. O., I. Elbatal, and S. Huang. 2016. Beta Linear Failure Rate Geometric Distribution with Applications. Journal of Data Science 14 (2):317.

[22] Pena-Ramirez, F. A., R. R. Guerra, G. M. Cordeiro, and P. R. Marinho. 2018. The Exponentiated Power Generalized Weibull: Properties and Applications. Anais da Academia Brasileira de Ciências 90 (3):2553-2577.

[23] Rinne, H. 2008. The Weibull distribution: a handbook: Chapman and Hall/CRC.

[24] Selim, M. A. 2018. The Generalized Power Generalized Weibull Distribution: Properties and Applications. arXiv preprint arXiv:1807.10763.

[25] Selim, M. A., and A. M. Badr. 2016. The Kumaraswamy generalized power Weibull distribution. Math. Theo. Model 6:110-124.

[26] Tahir, M., G. M. Cordeiro, S. Ali, S. Dey, and A. Manzoor. 2018. The inverted Nadarajah-Haghighi distribution: estimation methods and applications. Journal of Statistical Computation and Simulation:1-24.

[27] Team, R. C. 2015. R: A language and environment for statistical computing.

[28] Voda, V. G. 1972. On the inverse Rayleigh distributed random variable. Reports of Statistical Application Research 19:13-21. 